STRUCTURE REPORTS

ISSN 1600-5368

Received 6 August 2014

Accepted 30 September 2014

Edited by M. Zeller, Youngstown State University, USA

Keywords: crystal structure; hydrogen bonds; $\pi-\pi$ stacking; $\mathrm{Ni}^{\mathrm{I}}-\mathrm{Na}$ heterometallic complex; Schiff base

CCDC reference: 1026857

Supporting information: this article has

supporting information at journals.iucr.org/e

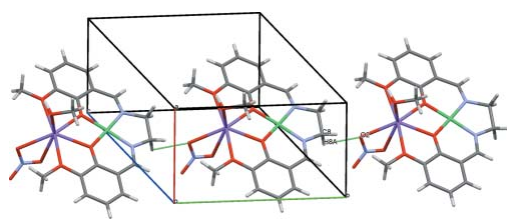

OPEN $\curvearrowright$ ACCESS

\section{Crystal structure of $\left\{\boldsymbol{\mu}-6, \mathbf{6}^{\prime}\right.$-dimethoxy-2, $\mathbf{2}^{\prime}$-[ethane- 1,2-diylbis(nitrilomethanylylidene)]diphenolato\}- (methanol)(nitrato)nickel(II)sodium}

\author{
Olesia V. Moroz, ${ }^{a *}$ Viktor A. Trush, ${ }^{a}$ Tatiana Yu. Sliva, ${ }^{a}$ Irina S. Konovalova ${ }^{b}$ and \\ Vladimir M. Amirkhanov ${ }^{\mathrm{a}}$
}

${ }^{a}$ Taras Shevchenko National University of Kyiv, Department of Chemistry, 64/13 Volodymyrska Street, Kyiv 01601,

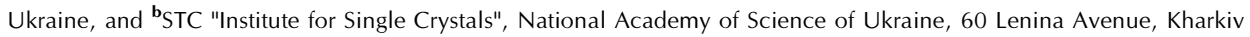
61001, Ukraine.*Correspondence e-mail: ovmoroz@yahoo.com

In the molecular structure of the title compound, $\left[\mathrm{NaNi}\left(\mathrm{C}_{18} \mathrm{H}_{18} \mathrm{~N}_{2} \mathrm{O}_{4}\right)\left(\mathrm{NO}_{3}\right)(-\right.$ $\left.\mathrm{CH}_{3} \mathrm{OH}\right)$, the $\mathrm{Ni}^{2+}$ ion has a slightly distorted square-planar coordination environment defined by two $\mathrm{N}$ and two $\mathrm{O}$ atoms which belong to a Schiff base ligand, viz. 6,6'-dimethoxy-2,2'-[ethane-1,2-diylbis(nitrilomethanylylidene)]diphenolate. Seven $\mathrm{O}$ atoms form the coordination environment of the $\mathrm{Na}^{+}$ion: four from the Schiff base ligand, two from a bidentate chelating nitrate anion and one $\mathrm{O}$ atom from a coordinating methanol molecule. In the crystal, the bimetallic complexes are assembled into chains along the $b$-axis direction via weak $\mathrm{C}-\mathrm{H}$... O hydrogen-bond interactions. Neighbouring chains are in turn connected through bifurcated $\mathrm{O}-\mathrm{H} \cdots \mathrm{O}$ hydrogen bonds that involve the coordinating methanol molecules and the nitrate anions, and through $\pi-\pi$ stacking interactions between phenyl rings of neighbouring molecules.

\section{Chemical context}

Schiff bases are known to be effective ligands able to coordinate a wide range of different metal ions, and they have been widely utilized in the study of biochemical processes (Lindoy et al., 1976; Correia et al., 2005). Compartmental Schiff base ligands, i.e. tetra- and hexadentate Schiff base ligands with different 'compartments' for different types of metal ions, have been employed extensively as 'blocking ligands'. Typical examples would be e.g. ligands with an $\mathrm{N}_{2} \mathrm{O}_{4}$ donor set with two Schiff base N-donor sites, two anionic phenolate donor sites, and two additional ether donor sites. The $\mathrm{N}_{2} \mathrm{O}_{2}$ compartment is generally more favorable for $3 d$ metal ions. The additional O-donor atoms provide the opportunity to accommodate a second metal ion, which might be a $3 d-, 4 f-, s-$ or $p$-block element, thus allowing the production of di-, tri- or oligonuclear systems (Gheorghe et al., 2006; Costes et al., 2008; Andruh et al., 2009).

Studies on heterometallic complexes began at the end of the 1960s. They are of interest because of their physicochemical properties that arise from the presence of dissimilar metal ions in close proximity. The majority of publications in this field are devoted to the preparation of $3 d-4 f$ heterometallic complexes (Costes et al., 1998; Koner et al., 2005; Sakamoto et al., 2001). Metal salicylaldimines, on the other hand, represent a fascinating group of ligands that are not only effective complexing agents for $p$ - and $d$-block elements, but also for alkali metal ions similar to the more well known ligand systems such as crown ethers, cryptands etc. Much of the interest concerning 
the coordination chemistry of alkali metal ions originates from the development of molecular systems that can mimic naturally occurring molecules that are responsible for the selective transport of these ions, e.g. through membranes. Some of the alkali-metal-ion adducts behave as precursors for other potentially interesting molecular species that can be used for small-molecule activation (Gambarotta et al., 1982), electron storage (Gallo et al., 1997) and the production of materials with remarkable magnetic properties, the alkali cation being crucial in determining the three-dimensional network in the solid state (Miyasaka et al., 1996).

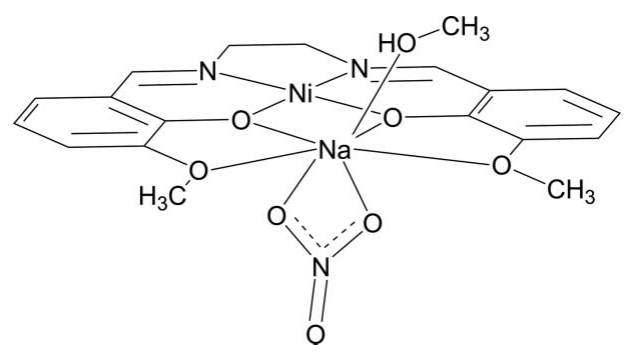

In the case of compartmental Schiff base ligands such as $e . g$. $\mathrm{N}$ (imine $)_{2} \mathrm{O}$ (phenoxo $)_{2} \mathrm{O}$ (methoxy/ethoxy $)_{2}$, the metal ion may be either retained in the plane of the $\mathrm{O}_{4}$ donor set or sandwiched between two sets of the Schiff base $\mathrm{O}$ atoms. The former case is usually characterized by a coordination number of eight from two $\mathrm{O}$ (phenoxo $)_{2} \mathrm{O}$ (methoxy/ethoxy $)_{2}$ compartments which belong to different molecules. The latter features a coordination number of six from the $\mathrm{O}_{4}$ compartment of the Schiff base, and two other donors are provided by coordinating solvent molecules and/or anions. The present paper is devoted to the synthesis and structural analysis of an $\mathrm{Ni}^{2+}$-containing complex $\left[\mathrm{NaNi}(L)\left(\mathrm{CH}_{3} \mathrm{OH}\right)\left(\mathrm{NO}_{3}\right)\right]$, (I), in which the $\mathrm{Na}^{+}$ion has a seven-coordination geometry and where $\mathrm{H}_{2} L$ is the compartmental Schiff base ligand 6,6'dimethoxy-2,2' -(ethane-1,2-diyldiiminodimethylene)diphenol.

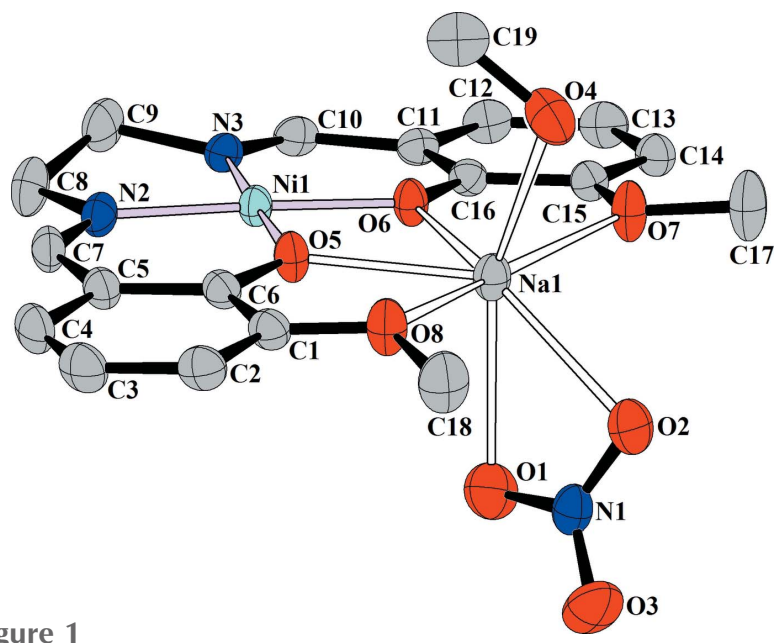

The molecular structure of (I), showing $30 \%$ probability displacement ellipsoids and the atom-numbering scheme. $\mathrm{H}$ atoms have been omitted for clarity.
Table 1

Hydrogen-bond geometry $\left(\AA{ }^{\circ}\right)$.

\begin{tabular}{lllll}
\hline$D-\mathrm{H} \cdots A$ & $D-\mathrm{H}$ & $\mathrm{H} \cdots A$ & $D \cdots A$ & $D-\mathrm{H} \cdots A$ \\
\hline $\mathrm{O} 4-\mathrm{H} 4 O \cdots \mathrm{O}^{\mathrm{i}}$ & 0.82 & 2.24 & $2.991(2)$ & 154 \\
$\mathrm{O}^{\mathrm{i}}-\mathrm{H} 4 O \cdots{ }^{\mathrm{i}}$ & 0.82 & 2.49 & $3.181(2)$ & 143 \\
$\mathrm{C} 8-\mathrm{H} 8 B \cdots 2^{\mathrm{ii}}$ & 0.97 & 2.65 & $3.152(2)$ & 112 \\
\hline
\end{tabular}

Symmetry codes: (i) $x-1, y, z$; (ii) $x, y-1, z$.

\section{Structural commentary}

The molecular structure of compound (I) with the atom numbering is shown in Fig. 1. Two phenolate $\mathrm{O}$ atoms provided by the Schiff base ligand create a double bridge between the $\mathrm{Ni}^{2+}$ and $\mathrm{Na}^{+}$ions. The coordination environment of the $\mathrm{Ni}^{2+}$ ion is square-planar, formed by two imine $\mathrm{N}$ atoms and two phenolate $\mathrm{O}$ atoms. The $\mathrm{Na}^{+}$ion has an unusual seven-coordinated geometry in which the ion sits in the plane of the Schiff base $\mathrm{O}$ atoms. Further significant interactions with two nitrate $\mathrm{O}$ atoms and one $\mathrm{O}$ atom from the coordinating methanol molecule, which are located above and below the plane formed by $L$, complete the coordination sphere. Values for the geometric parameters in (I) are in good agreement with those observed for complexes based on similar Schiff base ligands (Allen et al., 1987; Cunningham et al., 2000; Wang \& Shen, 2009; Xiao, 2009). The two phenoxo and two methoxy $\mathrm{O}$ atoms of the $\mathrm{O}$ (phenoxo $)_{2} \mathrm{O}(\text { ethoxy })_{2}$ moiety adopt a planar geometry as evidenced by the small mean deviation of the $\mathrm{O}$ atoms $(<0.02 \AA)$, from the 05/06/O7/O8 least-squares plane. The deviations of the $\mathrm{Na}^{+}$and $\mathrm{Ni}^{2+}$ ions from the $05 / 06 / 07 / 08$ plane $[0.166(1)$ and 0.008 (2) $\AA$, respectively] indicate that $\mathrm{Na}$ and $\mathrm{Ni}$ are well incorporated in the $\mathrm{O}$ (phenoxo $)_{2} \mathrm{O}$ (ethoxy $)_{2}$ moiety.

\section{Supramolecular features}

In the crystal structure, the molecules of the title compound form chains along the $b$-axis via weak $\mathrm{C}-\mathrm{H} \cdots \mathrm{O}$ hydrogenbond interactions (Fig. 2, Table 1). The $\mathrm{C}$ atom of the ethylene moiety acts as a donor and one $\mathrm{O}$ atom of the nitrate anion of the neighboring molecule acts as an acceptor. These chains are further assembled into sheets by a bifurcated $\mathrm{O}-\mathrm{H} \cdots \mathrm{O}$ hydrogen bond (Steiner, 2002), which involves the coordin-

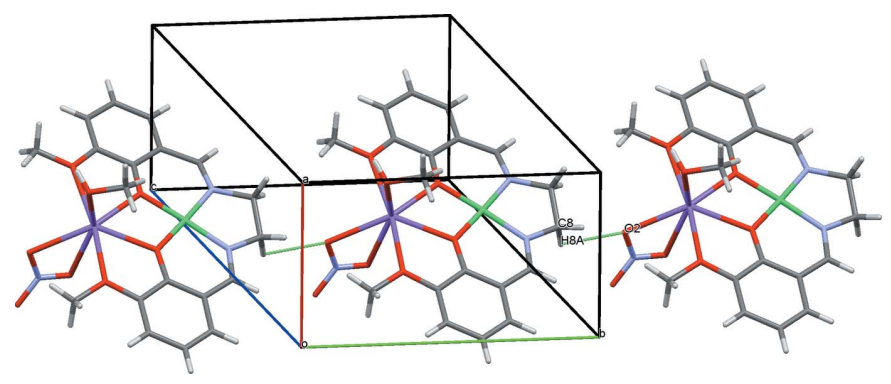

Figure 2

The molecular packing for (I), viewed along the $b$ axis. $\mathrm{C}-\mathrm{H} \cdots \mathrm{O}$ interactions are shown as dashed lines. 
Table 2

Experimental details.

\begin{tabular}{|c|c|}
\hline \multicolumn{2}{|l|}{ Crystal data } \\
\hline Chemical formula & $\begin{array}{l}{\left[\mathrm{NaNi}\left(\mathrm{C}_{18} \mathrm{H}_{18} \mathrm{~N}_{2} \mathrm{O}_{4}\right)\left(\mathrm{NO}_{3}\right)^{-}\right.} \\
\left.\quad\left(\mathrm{CH}_{4} \mathrm{O}\right)\right]\end{array}$ \\
\hline$M_{\mathrm{r}}$ & 502.09 \\
\hline Crystal system, space group & Triclinic, $P \overline{1}$ \\
\hline Temperature (K) & 293 \\
\hline$a, b, c(\AA)$ & $7.207(1), 11.047(1), 13.619(1)$ \\
\hline$\alpha, \beta, \gamma\left({ }^{\circ}\right)$ & $95.30(1), 99.81(1), 99.05(1)$ \\
\hline$V\left(\AA^{3}\right)$ & $1047.2(2)$ \\
\hline$Z$ & 2 \\
\hline Radiation type & Мо $K \alpha$ \\
\hline$\mu\left(\mathrm{mm}^{-1}\right)$ & 1.00 \\
\hline Crystal size (mm) & $0.4 \times 0.2 \times 0.2$ \\
\hline \multicolumn{2}{|l|}{ Data collection } \\
\hline Diffractometer & Nonius KappaCCD \\
\hline Absorption correction & $\begin{array}{l}\text { Multi-scan (SADABS; Sheldrick, } \\
\text { 2003) }\end{array}$ \\
\hline$T_{\min }, T_{\max }$ & $0.690,0.825$ \\
\hline $\begin{array}{l}\text { No. of measured, independent and } \\
\text { observed }[I>2 \sigma(I)] \text { reflections }\end{array}$ & $12718,6501,4324$ \\
\hline$R_{\text {int }}$ & 0.020 \\
\hline$(\sin \theta / \lambda)_{\max }\left(\AA^{-1}\right)$ & 0.744 \\
\hline \multicolumn{2}{|l|}{ Refinement } \\
\hline$R\left[F^{2}>2 \sigma\left(F^{2}\right)\right], w R\left(F^{2}\right), S$ & $0.035,0.081,0.90$ \\
\hline No. of reflections & 6501 \\
\hline No. of parameters & 292 \\
\hline $\mathrm{H}$-atom treatment & H-atom parameters constrained \\
\hline$\Delta \rho_{\max }, \Delta \rho_{\min }\left(\mathrm{e} \AA^{-3}\right)$ & $0.50,-0.32$ \\
\hline
\end{tabular}

Computer programs: COLLECT (Nonius, 1999), DENZO/SCALEPACK (Otwinowski \& Minor, 1997), SHELXS97 and SHELXL2014 (Sheldrick, 2008), ORTEP-3 for Windows and Win GX (Farrugia, 2012).

ating methanol molecule and nitrate units (Fig. 3, Table 1) and through $\pi-\pi$ stacking interactions, which exist between phenyl rings of neighbouring molecules, with a separation of 3.5845 (11) $\AA$ between the centroids formed by the $\mathrm{C}$ atoms of the rings [symmetry code: (iii) $-x+1,-y,-z$ ]. For the $\mathrm{O}-$ $\mathrm{H}$... O hydrogen bond, the $\mathrm{O}$ atom of the methanol molecule acts as a donor and the $\mathrm{O}$ atoms of the nitrate anion of the neighbouring molecule act as the acceptors.

\section{Synthesis and crystallization}

A mixture of 6,6'-dimethoxy-2,2'-(ethane-1,2-diyldiiminodimethylene)diphenol $(1 \mathrm{mmol})$ and nickel nitrate $(1 \mathrm{mmol})$ in methanol $(15 \mathrm{ml})$ was stirred for $30 \mathrm{~min}$ at room temperature. Then, sodium nitrate $(1 \mathrm{mmol})$ was added, and the mixture was stirred for another $30 \mathrm{~min}$ and filtered. The resulting clear orange filtrate was left at ambient temperature for crystallization in air. The red-orange block-shaped crystals were collected by filtration after $6 \mathrm{~d}$, washed with chilled isopropanol and dried on filter paper (yield $0.28 \mathrm{~g}, 56 \%$ ).

\section{Refinement}

$\mathrm{H}$ atoms were placed in geometrically idealized positions and constrained to ride on their parent atoms, with $\mathrm{C}-\mathrm{H}$ distances of 0.95 (aromatic) or $0.99 \AA$ (methylene), with $U_{\text {iso }}(\mathrm{H})=$ $1.2 U_{\text {eq }}(\mathrm{C}), \mathrm{C}-\mathrm{H}=0.98 \AA$ for methyl $\mathrm{H}$ atoms, with $U_{\text {iso }}(\mathrm{H})=$

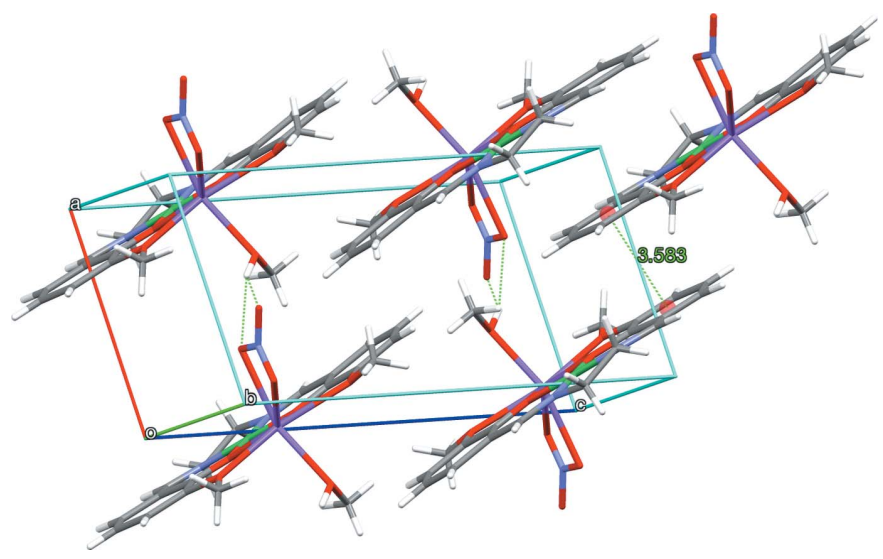

Figure 3

$\mathrm{O}-\mathrm{H} \cdots \mathrm{O}$ and $\pi-\pi$ contacts for (I), shown as dashed lines, with ring centroids shown as coloured spheres.

$1.5 U_{\mathrm{eq}}(\mathrm{C})$, and $\mathrm{O}-\mathrm{H}=0.82 \AA$ for the hydroxy group of methanol, with $U_{\text {iso }}(\mathrm{H})=1.5 U_{\mathrm{eq}}(\mathrm{O})$. Crystal data, data collection and structure refinement details are summarized in Table 2.

\section{Acknowledgements}

The authors are grateful to Dr Y. S. Moroz for his kind assistance in refining the structure.

\section{References}

Allen, F. H., Kennard, O., Watson, D. G., Brammer, L., Orpen, A. G. \& Taylor, R. (1987). J. Chem. Soc. Perkin Trans. 2, pp. S1-19.

Andruh, M., Branzea, D. G., Gheorghe, R. \& Madalan, A. M. (2009). CrystEngComm, 11, 2571-2584.

Correia, I., Pessoa, J. C., Duarte, M. T., da Piedade, M. F. M., Jackush, T., Kiss, T., Castro, M. M. C. A., Geraldes, C. F. C. G. \& Avecilla, F. (2005). Eur. J. Inorg. Chem. pp. 732-744.

Costes, J.-P., Dahan, F., Dupuis, A. \& Laurent, J.-P. (1998). Chem. Eur. J. 4, 1616-1620.

Costes, J.-P., Shova, S. \& Wernsdorfer, W. (2008). Dalton Trans. pp. 1843-1849.

Cunningham, D., McArdle, P., Mitchell, M., Chonchubhair, N. N., O'Gara, M., Franceschi, F. \& Floriani, C. (2000). Inorg. Chem. 39, 1639-1649.

Farrugia, L. J. (2012). J. Appl. Cryst. 45, 849-854.

Gallo, E., Solari, E., Re, N., Floriani, C., Chiesi-Villa, A. \& Rizzoli, C. (1997). J. Am. Chem. Soc. 119, 5144-5154.

Gambarotta, S., Arena, F., Floriani, C. \& Zanazzi, P. F. (1982). J. Am. Chem. Soc. 104, 5082-5092.

Gheorghe, R., Cucos, P., Andruh, M., Costes, J.-P., Donnadieu, B. \& Shova, S. (2006). Chem. Eur. J. 12, 187-203.

Koner, R., Lin, H.-H., Wei, H.-H. \& Mohanta, S. (2005). Inorg. Chem. 44, 3524-3536.

Lindoy, L. F., Lip, H. C., Power, L. F. \& Rea, T. H. (1976). Inorg. Chem. 15, 1724-1727.

Miyasaka, H., Matsumoto, N., Ōkawa, H., Re, N., Gallo, E. \& Floriani, C. (1996). J. Am. Chem. Soc. 118, 981-994.

Nonius (1999). COLLECT. Nonius BV, Delft, The Netherlands.

Otwinowski, Z. \& Minor, W. (1997). Methods in Enzymology, Vol. 276, Macromolecular Crystallography, Part A, edited by C. W. Carter Jr \& R. M. Sweet, pp. 307-326. New York: Academic Press. Sakamoto, M., Manseki, K. \& Okawa, H. (2001). Coord. Chem. Rev. 219-221, 379-414. 
Sheldrick, G. M. (2003). SADABS. University of Göttingen, Germany.

Sheldrick, G. M. (2008). Acta Cryst. A64, 112-122.
Steiner, T. (2002). Angew. Chem. Int. Ed. 41, 48-76.

Wang, W. \& Shen, Y.-M. (2009). Acta Cryst. E65, m557.

Xiao, H.-Q. (2009). Acta Cryst. E65, m400. 


\section{supporting information}

Acta Cryst. (2014). E70, 305-308 [doi:10.1107/S160053681402159X]

\section{Crystal structure of $\left\{\mu-6,6^{\prime}\right.$-dimethoxy-2,2'-[ethane-1,2-diylbis(nitrilo- methanylylidene)]diphenolato\}(methanol)(nitrato)nickel(II)sodium}

\section{Olesia V. Moroz, Viktor A. Trush, Tatiana Yu. Sliva, Irina S. Konovalova and Vladimir M.} Amirkhanov

\section{Computing details}

Data collection: COLLECT (Nonius, 1999); cell refinement: DENZO/SCALEPACK (Otwinowski \& Minor, 1997); data reduction: DENZO/SCALEPACK (Otwinowski \& Minor, 1997); program(s) used to solve structure: SHELXS97

(Sheldrick, 2008); program(s) used to refine structure: SHELXL2014 (Sheldrick, 2008); molecular graphics: ORTEP-3 for Windows (Farrugia, 2012); software used to prepare material for publication: Win GX (Farrugia, 2012).

$\{\mu$-6,6'-Dimethoxy-2,2'-[ethane-1,2-diylbis(nitrilomethanylylidene)]diphenolato\}(methanol) (nitrato)nickel(II)sodium

Crystal data

$\left[\mathrm{NaNi}\left(\mathrm{C}_{18} \mathrm{H}_{18} \mathrm{~N}_{2} \mathrm{O}_{4}\right)\left(\mathrm{NO}_{3}\right)\left(\mathrm{CH}_{4} \mathrm{O}\right)\right]$

$M_{r}=502.09$

Triclinic, $P \overline{1}$

$a=7.207(1) \AA$

$b=11.047(1) \AA$

$c=13.619(1) \AA$

$\alpha=95.30(1)^{\circ}$

$\beta=99.81(1)^{\circ}$

$\gamma=99.05(1)^{\circ}$

$V=1047.2(2) \AA^{3}$

Data collection

Nonius KappaCCD diffractometer

Radiation source: sealed X-ray tube $\varphi$ scans and $\omega$ scans with $\kappa$ offset Absorption correction: multi-scan (SADABS; Sheldrick, 2003)

$T_{\text {min }}=0.690, T_{\text {max }}=0.825$

12718 measured reflections

Refinement

Refinement on $F^{2}$

Least-squares matrix: full

$R\left[F^{2}>2 \sigma\left(F^{2}\right)\right]=0.035$

$w R\left(F^{2}\right)=0.081$

$S=0.90$

6501 reflections
$Z=2$

$F(000)=520$

$D_{\mathrm{x}}=1.592 \mathrm{Mg} \mathrm{m}^{-3}$

Mo $K \alpha$ radiation, $\lambda=0.71073 \AA$

Cell parameters from 12718 reflections

$\theta=2.9-31.9^{\circ}$

$\mu=1.00 \mathrm{~mm}^{-1}$

$T=293 \mathrm{~K}$

Block, white

$0.4 \times 0.2 \times 0.2 \mathrm{~mm}$

6501 independent reflections 4324 reflections with $I>2 \sigma(I)$

$R_{\text {int }}=0.020$

$\theta_{\text {max }}=31.9^{\circ}, \theta_{\min }=2.9^{\circ}$

$h=-8 \rightarrow 10$

$k=-15 \rightarrow 15$

$l=-19 \rightarrow 20$

292 parameters

0 restraints

Hydrogen site location: mixed

$\mathrm{H}$-atom parameters constrained

$w=1 /\left[\sigma^{2}\left(F_{\mathrm{o}}{ }^{2}\right)+(0.0431 P)^{2}\right]$

where $P=\left(F_{\mathrm{o}}^{2}+2 F_{\mathrm{c}}^{2}\right) / 3$ 
$(\Delta / \sigma)_{\max }<0.001$

$\Delta \rho_{\max }=0.50$ e $\AA^{-3}$

Special details

Geometry. All e.s.d.'s (except the e.s.d. in the dihedral angle between two 1.s. planes) are estimated using the full covariance matrix. The cell e.s.d.'s are taken into account individually in the estimation of e.s.d.'s in distances, angles and torsion angles; correlations between e.s.d.'s in cell parameters are only used when they are defined by crystal symmetry. An approximate (isotropic) treatment of cell e.s.d.'s is used for estimating e.s.d.'s involving 1.s. planes.

Refinement. Refinement of $F^{2}$ against ALL reflections. The weighted $R$-factor $w R$ and goodness of fit $S$ are based on $F^{2}$, conventional $R$-factors $R$ are based on $F$, with $F$ set to zero for negative $F^{2}$. The threshold expression of $F^{2}>\sigma\left(F^{2}\right)$ is used only for calculating $R$-factors(gt) etc. and is not relevant to the choice of reflections for refinement. $R$-factors based on $F^{2}$ are statistically about twice as large as those based on $F$, and $R$ - factors based on ALL data will be even larger.

Fractional atomic coordinates and isotropic or equivalent isotropic displacement parameters $\left(\AA^{2}\right)$

\begin{tabular}{|c|c|c|c|c|}
\hline & $x$ & $y$ & $z$ & $U_{\text {iso }} * / U_{\text {eq }}$ \\
\hline N1 & $1.3085(3)$ & $0.47914(14)$ & $0.20473(11)$ & $0.0500(4)$ \\
\hline $\mathrm{N} 2$ & $1.0634(2)$ & $-0.10231(12)$ & $0.31510(10)$ & $0.0414(3)$ \\
\hline N3 & 0.87587 (19) & $-0.13606(11)$ & $0.13663(10)$ & $0.0384(3)$ \\
\hline $\mathrm{O} 1$ & $1.3115(2)$ & $0.36716(13)$ & $0.19219(14)$ & $0.0823(5)$ \\
\hline $\mathrm{O} 2$ & $1.1514(2)$ & $0.51204(13)$ & $0.20629(12)$ & $0.0692(4)$ \\
\hline $\mathrm{O} 3$ & $1.4575(2)$ & $0.55470(16)$ & $0.21536(12)$ & $0.0840(5)$ \\
\hline $\mathrm{O} 4$ & $0.7094(2)$ & $0.36010(15)$ & $0.29641(10)$ & $0.0708(4)$ \\
\hline $\mathrm{H} 4 \mathrm{O}$ & 0.6210 & 0.3784 & 0.2582 & $0.106^{*}$ \\
\hline O5 & $1.04924(18)$ & $0.14171(10)$ & $0.31975(8)$ & $0.0439(3)$ \\
\hline O6 & $0.87634(17)$ & $0.10986(10)$ & $0.14652(8)$ & $0.0401(3)$ \\
\hline $\mathrm{O} 7$ & $0.7790(2)$ & $0.29628(11)$ & $0.06339(9)$ & $0.0531(3)$ \\
\hline $\mathrm{O} 8$ & $1.1398(2)$ & $0.36213(11)$ & $0.41444(9)$ & $0.0539(3)$ \\
\hline $\mathrm{C} 1$ & $1.2043(2)$ & $0.27108(16)$ & $0.46557(12)$ & $0.0417(4)$ \\
\hline $\mathrm{C} 2$ & $1.3145(3)$ & 0.28976 (19) & $0.56084(13)$ & $0.0504(4)$ \\
\hline $\mathrm{H} 2$ & 1.3492 & 0.3688 & 0.5958 & $0.060 *$ \\
\hline $\mathrm{C} 3$ & $1.3734(3)$ & $0.1895(2)$ & $0.60439(14)$ & $0.0592(5)$ \\
\hline H3 & 1.4487 & 0.2022 & 0.6683 & $0.071^{*}$ \\
\hline $\mathrm{C} 4$ & $1.3227(3)$ & $0.0743(2)$ & $0.55493(13)$ & $0.0554(5)$ \\
\hline $\mathrm{H} 4$ & 1.3625 & 0.0085 & 0.5856 & $0.067^{*}$ \\
\hline $\mathrm{C} 5$ & $1.2091(2)$ & $0.05142(16)$ & $0.45629(12)$ & 0.0419 (4) \\
\hline C6 & $1.1504(2)$ & $0.15157(15)$ & $0.41092(11)$ & $0.0380(4)$ \\
\hline $\mathrm{C} 7$ & $1.1604(3)$ & $-0.07053(17)$ & $0.40535(13)$ & 0.0457 (4) \\
\hline $\mathrm{H} 7$ & 1.2019 & -0.1330 & 0.4399 & $0.055^{*}$ \\
\hline $\mathrm{C} 8$ & $1.0406(3)$ & $-0.23159(17)$ & $0.27149(14)$ & $0.0585(5)$ \\
\hline $\mathrm{H} 8 \mathrm{~A}$ & 1.0011 & -0.2860 & 0.3189 & $0.070^{*}$ \\
\hline H8B & 1.1616 & -0.2488 & 0.2568 & $0.070^{*}$ \\
\hline C9 & $0.8924(3)$ & $-0.25411(15)$ & $0.17663(15)$ & $0.0578(5)$ \\
\hline H9A & 0.9287 & -0.3105 & 0.1274 & $0.069^{*}$ \\
\hline H9B & 0.7699 & -0.2914 & 0.1905 & $0.069^{*}$ \\
\hline $\mathrm{C} 10$ & $0.7997(2)$ & $-0.13762(15)$ & $0.04360(13)$ & $0.0411(4)$ \\
\hline H10 & 0.7711 & -0.2136 & 0.0037 & $0.049^{*}$ \\
\hline C11 & $0.7552(2)$ & $-0.03232(15)$ & $-0.00347(11)$ & $0.0366(3)$ \\
\hline $\mathrm{C} 12$ & $0.6631(3)$ & $-0.04768(17)$ & $-0.10544(12)$ & $0.0455(4)$ \\
\hline
\end{tabular}




$\begin{array}{lllll}\text { H12 } & 0.6384 & -0.1255 & -0.1424 & 0.055^{*} \\ \text { C13 } & 0.6106(3) & 0.04949(18) & -0.14969(12) & 0.0489(4) \\ \text { H13 } & 0.5500 & 0.0374 & -0.2167 & 0.059^{*} \\ \text { C14 } & 0.6459(2) & 0.16813(16) & -0.09638(12) & 0.0419(4) \\ \text { H14 } & 0.6087 & 0.2342 & -0.1276 & 0.050^{*} \\ \text { C15 } & 0.7366(2) & 0.18574(15) & 0.00292(11) & 0.0372(4) \\ \text { C16 } & 0.7923(2) & 0.08597(14) & 0.05155(11) & 0.0340(3) \\ \text { C17 } & 0.7114(4) & 0.39946(18) & 0.02595(15) & 0.0719(7) \\ \text { H17A } & 0.7727 & 0.4218 & -0.0287 & 0.108^{*} \\ \text { H17B } & 0.7399 & 0.4676 & 0.0784 & 0.108^{*} \\ \text { H17C } & 0.5755 & 0.3791 & 0.0027 & 0.108^{*} \\ \text { C18 } & 1.2117(4) & 0.48674(18) & 0.45544(16) & 0.0714(7) \\ \text { H18A } & 1.1842 & 0.5002 & 0.5217 & 0.107^{*} \\ \text { H18B } & 1.1519 & 0.5405 & 0.4136 & 0.107^{*} \\ \text { H18C } & 1.3475 & 0.5039 & 0.4587 & 0.107^{*} \\ \text { C19 } & 0.6357(4) & 0.2606(2) & 0.34481(17) & 0.0756(7) \\ \text { H19A } & 0.6104 & 0.1856 & 0.2994 & 0.113^{*} \\ \text { H19B } & 0.5192 & 0.2755 & 0.3650 & 0.113^{*} \\ \text { H19C } & 0.7275 & 0.2531 & 0.4029 & 0.113^{*} \\ \text { Ni1 } & 0.96567(3) & 0.00143(2) & 0.22924(2) & 0.03524(7) \\ \text { Na1 } & 0.97774(10) & 0.30835(6) & 0.23162(5) & 0.04288(16)\end{array}$

Atomic displacement parameters $\left(\AA^{2}\right)$

\begin{tabular}{|c|c|c|c|c|c|c|}
\hline & $U^{11}$ & $U^{22}$ & $U^{33}$ & $U^{12}$ & $U^{13}$ & $U^{23}$ \\
\hline N1 & $0.0603(11)$ & $0.0439(9)$ & $0.0431(8)$ & $0.0079(8)$ & $0.0013(7)$ & $0.0085(7)$ \\
\hline N2 & $0.0517(9)$ & $0.0324(7)$ & $0.0464(8)$ & $0.0153(6)$ & $0.0169(7)$ & $0.0103(6)$ \\
\hline N3 & $0.0379(8)$ & $0.0283(7)$ & $0.0493(8)$ & 0.0060 & $0.0091(6)$ & $0.0051(6)$ \\
\hline $\mathrm{O} 1$ & $0.0755(12)$ & $0.0449(9)$ & $0.1300(14)$ & $0.0208(8)$ & $0.0217(10)$ & $0.0081(9)$ \\
\hline $\mathrm{O} 2$ & $0.0728(11)$ & $0.0523(9)$ & 0.0915 (11) & $0.0276(8)$ & $0.0203(8)$ & $0.0175(7)$ \\
\hline $\mathrm{O} 3$ & $0.0702(12)$ & $0.0779(11)$ & $0.0851(11)$ & $-0.0239(9)$ & $-0.0063(8)$ & $0.0156(9)$ \\
\hline $\mathrm{O} 4$ & $0.0659(10)$ & $0.0912(11)$ & $0.0610(9)$ & $0.0337(9)$ & $0.0065(7)$ & $0.0143(8)$ \\
\hline $\mathrm{O} 5$ & $0.0570(8)$ & $0.0342(6)$ & $0.0367(6)$ & $0.0132(5)$ & $-0.0063(5)$ & $0.0041(5)$ \\
\hline O6 & $0.0512(7)$ & $0.0295(6)$ & $0.0350(6)$ & $0.0094(5)$ & $-0.0055(5)$ & $0.0019(4)$ \\
\hline $\mathrm{O} 7$ & $0.0809(10)$ & $0.0335(6)$ & $0.0400(6)$ & $0.0180(6)$ & $-0.0087(6)$ & $0.0025(5)$ \\
\hline O8 & $0.0715(9)$ & $0.0379(7)$ & $0.0434(7)$ & $0.0095(6)$ & $-0.0106(6)$ & $-0.0001(5)$ \\
\hline $\mathrm{C} 1$ & $0.0384(10)$ & $0.0475(10)$ & $0.0373(8)$ & $0.0068(7)$ & $0.0027(7)$ & $0.0043(7)$ \\
\hline $\mathrm{C} 2$ & $0.0466(11)$ & $0.0605(12)$ & $0.0384(9)$ & $0.0031(9)$ & $0.0000(8)$ & $0.0025(8)$ \\
\hline $\mathrm{C} 3$ & 0.0523 & $0.0792(15)$ & $0.0418(10)$ & $0.0118(10)$ & $-0.0052(8)$ & $0.0112(10)$ \\
\hline $\mathrm{C} 4$ & $0.0549(13)$ & $0.0717(14)$ & $0.0440(10)$ & $0.0226(10)$ & $0.0022(8)$ & $0.0227(9)$ \\
\hline $\mathrm{C} 5$ & $0.0403(10)$ & $0.0495(10)$ & $0.0397(9)$ & $0.0145(8)$ & $0.0076(7)$ & $0.0131(7)$ \\
\hline C6 & $0.0339(9)$ & $0.0467(10)$ & $0.0344(8)$ & $0.0092(7)$ & $0.0049(6)$ & $0.0089(7)$ \\
\hline $\mathrm{C} 7$ & $0.0502(11)$ & $0.0501(11)$ & $0.0467(10)$ & $0.0230(8)$ & $0.0145(8)$ & $0.0231(8)$ \\
\hline $\mathrm{C} 8$ & $0.0886(16)$ & $0.0380(10)$ & $0.0563(11)$ & $0.0250(10)$ & $0.0168(10)$ & $0.0146(8)$ \\
\hline C9 & $0.0685(14)$ & $0.0264(9)$ & $0.0742(13)$ & $0.0049(8)$ & $0.0038(10)$ & $0.0070(8)$ \\
\hline $\mathrm{C} 10$ & $0.0410(10)$ & $0.0290(8)$ & $0.0507(10)$ & $0.0018(7)$ & $0.0107(7)$ & $-0.0052(7)$ \\
\hline $\mathrm{C} 11$ & $0.0338(9)$ & $0.0360(8)$ & $0.0386(8)$ & $0.0031(6)$ & $0.0076(6)$ & $0.0006(6)$ \\
\hline $\mathrm{C} 12$ & $0.0492(11)$ & $0.0443(10)$ & $0.0379(9)$ & $0.0016(8)$ & $0.0075(7)$ & $-0.0079(7)$ \\
\hline
\end{tabular}


supporting information

\begin{tabular}{lllllll}
$\mathrm{C} 13$ & $0.0497(11)$ & $0.0615(12)$ & $0.0309(8)$ & $0.0046(9)$ & $0.0022(7)$ & $0.0003(8)$ \\
$\mathrm{C} 14$ & $0.0421(10)$ & $0.0480(10)$ & $0.0357(8)$ & $0.0098(7)$ & $0.0044(7)$ & $0.0083(7)$ \\
$\mathrm{C} 15$ & $0.0378(9)$ & $0.0377(9)$ & $0.0352(8)$ & $0.0079(7)$ & $0.0036(6)$ & $0.0043(6)$ \\
$\mathrm{C} 16$ & $0.0326(9)$ & $0.0327(8)$ & $0.0346(8)$ & $0.0039(6)$ & $0.0041(6)$ & $0.0009(6)$ \\
$\mathrm{C} 17$ & $0.111(2)$ & $0.0414(11)$ & $0.0596(12)$ & $0.0280(11)$ & $-0.0082(12)$ & $0.0091(9)$ \\
$\mathrm{C} 18$ & $0.0865(18)$ & $0.0455(12)$ & $0.0671(13)$ & $0.0025(11)$ & $-0.0136(12)$ & $-0.0035(10)$ \\
$\mathrm{C} 19$ & $0.0713(17)$ & $0.0801(17)$ & $0.0719(15)$ & $0.0061(13)$ & $0.0154(12)$ & $0.0002(13)$ \\
$\mathrm{Ni} 1$ & $0.03972(13)$ & $0.02768(11)$ & $0.03891(12)$ & $0.00921(8)$ & $0.00475(8)$ & $0.00648(8)$ \\
$\mathrm{Na} 1$ & $0.0529(4)$ & $0.0312(3)$ & $0.0421(3)$ & $0.0085(3)$ & $0.0013(3)$ & $0.0039(3)$ \\
\hline
\end{tabular}

Geometric parameters $\left(\AA,{ }^{o}\right)$

\begin{tabular}{|c|c|c|c|}
\hline $\mathrm{N} 1-\mathrm{O} 3$ & $1.230(2)$ & $\mathrm{C} 4-\mathrm{C} 5$ & $1.427(2)$ \\
\hline $\mathrm{N} 1-\mathrm{O} 1$ & $1.2372(19)$ & $\mathrm{C} 4-\mathrm{H} 4$ & 0.9300 \\
\hline $\mathrm{N} 1-\mathrm{O} 2$ & $1.246(2)$ & $\mathrm{C} 5-\mathrm{C} 6$ & $1.403(2)$ \\
\hline $\mathrm{N} 1-\mathrm{Na} 1$ & $2.8961(19)$ & $\mathrm{C} 5-\mathrm{C} 7$ & $1.420(2)$ \\
\hline $\mathrm{N} 2-\mathrm{C} 7$ & $1.293(2)$ & $\mathrm{C} 7-\mathrm{H} 7$ & 0.9300 \\
\hline $\mathrm{N} 2-\mathrm{C} 8$ & $1.468(2)$ & $\mathrm{C} 8-\mathrm{C} 9$ & $1.503(3)$ \\
\hline $\mathrm{N} 2-\mathrm{Ni} 1$ & $1.8433(13)$ & $\mathrm{C} 8-\mathrm{H} 8 \mathrm{~A}$ & 0.9700 \\
\hline $\mathrm{N} 3-\mathrm{C} 10$ & $1.290(2)$ & $\mathrm{C} 8-\mathrm{H} 8 \mathrm{~B}$ & 0.9700 \\
\hline $\mathrm{N} 3-\mathrm{C} 9$ & $1.473(2)$ & C9-H9A & 0.9700 \\
\hline $\mathrm{N} 3-\mathrm{Ni1}$ & $1.8371(13)$ & C9-H9B & 0.9700 \\
\hline $\mathrm{O} 1-\mathrm{Na} 1$ & $2.5512(19)$ & $\mathrm{C} 10-\mathrm{C} 11$ & $1.432(2)$ \\
\hline $\mathrm{O} 2-\mathrm{Na} 1$ & $2.4806(16)$ & $\mathrm{C} 10-\mathrm{H} 10$ & 0.9300 \\
\hline $\mathrm{O} 4-\mathrm{C} 19$ & $1.412(3)$ & $\mathrm{C} 11-\mathrm{C} 16$ & $1.408(2)$ \\
\hline $\mathrm{O} 4-\mathrm{Na} 1$ & $2.3837(17)$ & $\mathrm{C} 11-\mathrm{C} 12$ & $1.416(2)$ \\
\hline $\mathrm{O} 4-\mathrm{H} 4 \mathrm{O}$ & 0.8151 & $\mathrm{C} 12-\mathrm{C} 13$ & $1.352(3)$ \\
\hline $\mathrm{O} 5-\mathrm{C} 6$ & $1.3139(18)$ & $\mathrm{C} 12-\mathrm{H} 12$ & 0.9300 \\
\hline $\mathrm{O} 5-\mathrm{Ni} 1$ & $1.8396(11)$ & $\mathrm{C} 13-\mathrm{C} 14$ & $1.402(2)$ \\
\hline $\mathrm{O} 5-\mathrm{Na} 1$ & $2.3644(12)$ & $\mathrm{C} 13-\mathrm{H} 13$ & 0.9300 \\
\hline $\mathrm{O} 6-\mathrm{C} 16$ & $1.3148(17)$ & $\mathrm{C} 14-\mathrm{C} 15$ & $1.380(2)$ \\
\hline $\mathrm{O} 6-\mathrm{Ni1}$ & $1.8339(10)$ & C14-H14 & 0.9300 \\
\hline $\mathrm{O} 6-\mathrm{Na} 1$ & $2.3288(12)$ & $\mathrm{C} 15-\mathrm{C} 16$ & $1.413(2)$ \\
\hline $\mathrm{O} 7-\mathrm{C} 15$ & $1.3689(19)$ & C17-H17A & 0.9600 \\
\hline $\mathrm{O} 7-\mathrm{C} 17$ & $1.412(2)$ & C17-H17B & 0.9600 \\
\hline $\mathrm{O} 7-\mathrm{Na} 1$ & $2.4666(13)$ & $\mathrm{C} 17-\mathrm{H} 17 \mathrm{C}$ & 0.9600 \\
\hline $\mathrm{O} 8-\mathrm{C} 1$ & $1.371(2)$ & $\mathrm{C} 18-\mathrm{H} 18 \mathrm{~A}$ & 0.9600 \\
\hline $\mathrm{O} 8-\mathrm{C} 18$ & $1.418(2)$ & C18-H18B & 0.9600 \\
\hline $\mathrm{O} 8-\mathrm{Na} 1$ & $2.5364(13)$ & $\mathrm{C} 18-\mathrm{H} 18 \mathrm{C}$ & 0.9600 \\
\hline $\mathrm{C} 1-\mathrm{C} 2$ & $1.380(2)$ & $\mathrm{C} 19-\mathrm{Na} 1$ & $3.125(3)$ \\
\hline $\mathrm{C} 1-\mathrm{C} 6$ & $1.416(2)$ & C19-H19A & 0.9600 \\
\hline $\mathrm{C} 2-\mathrm{C} 3$ & $1.394(3)$ & C19-H19B & 0.9600 \\
\hline $\mathrm{C} 2-\mathrm{H} 2$ & 0.9300 & $\mathrm{C} 19-\mathrm{H} 19 \mathrm{C}$ & 0.9600 \\
\hline $\mathrm{C} 3-\mathrm{C} 4$ & $1.349(3)$ & Ni1-Na1 & $3.3749(7)$ \\
\hline $\mathrm{C} 3-\mathrm{H} 3$ & 0.9300 & & \\
\hline $\mathrm{O} 3-\mathrm{N} 1-\mathrm{O} 1$ & $120.31(19)$ & $\mathrm{O} 6-\mathrm{C} 16-\mathrm{C} 11$ & $123.80(14)$ \\
\hline $\mathrm{O} 3-\mathrm{N} 1-\mathrm{O} 2$ & $121.67(18)$ & $\mathrm{O} 6-\mathrm{C} 16-\mathrm{C} 15$ & $117.42(13)$ \\
\hline
\end{tabular}




\begin{tabular}{|c|c|c|c|}
\hline $\mathrm{O} 1-\mathrm{N} 1-\mathrm{O} 2$ & $118.02(17)$ & $\mathrm{C} 11-\mathrm{C} 16-\mathrm{C} 15$ & $118.78(13)$ \\
\hline $\mathrm{O} 3-\mathrm{N} 1-\mathrm{Na} 1$ & $166.28(12)$ & $\mathrm{O} 7-\mathrm{C} 17-\mathrm{H} 17 \mathrm{~A}$ & 109.5 \\
\hline $\mathrm{O} 1-\mathrm{N} 1-\mathrm{Na} 1$ & $61.59(11)$ & $\mathrm{O} 7-\mathrm{C} 17-\mathrm{H} 17 \mathrm{~B}$ & 109.5 \\
\hline $\mathrm{O} 2-\mathrm{N} 1-\mathrm{Na} 1$ & $58.35(10)$ & $\mathrm{H} 17 \mathrm{~A}-\mathrm{C} 17-\mathrm{H} 17 \mathrm{~B}$ & 109.5 \\
\hline $\mathrm{C} 7-\mathrm{N} 2-\mathrm{C} 8$ & $118.06(14)$ & $\mathrm{O} 7-\mathrm{C} 17-\mathrm{H} 17 \mathrm{C}$ & 109.5 \\
\hline $\mathrm{C} 7-\mathrm{N} 2-\mathrm{Ni} 1$ & $126.55(12)$ & $\mathrm{H} 17 \mathrm{~A}-\mathrm{C} 17-\mathrm{H} 17 \mathrm{C}$ & 109.5 \\
\hline $\mathrm{C} 8-\mathrm{N} 2-\mathrm{Ni} 1$ & $115.16(11)$ & $\mathrm{H} 17 \mathrm{~B}-\mathrm{C} 17-\mathrm{H} 17 \mathrm{C}$ & 109.5 \\
\hline $\mathrm{C} 10-\mathrm{N} 3-\mathrm{C} 9$ & $118.99(14)$ & $\mathrm{O} 8-\mathrm{C} 18-\mathrm{H} 18 \mathrm{~A}$ & 109.5 \\
\hline $\mathrm{C} 10-\mathrm{N} 3-\mathrm{Ni1}$ & $126.49(11)$ & $\mathrm{O} 8-\mathrm{C} 18-\mathrm{H} 18 \mathrm{~B}$ & 109.5 \\
\hline $\mathrm{C} 9-\mathrm{N} 3-\mathrm{Ni} 1$ & $114.51(11)$ & $\mathrm{H} 18 \mathrm{~A}-\mathrm{C} 18-\mathrm{H} 18 \mathrm{~B}$ & 109.5 \\
\hline $\mathrm{N} 1-\mathrm{O} 1-\mathrm{Na} 1$ & $93.16(12)$ & $\mathrm{O} 8-\mathrm{C} 18-\mathrm{H} 18 \mathrm{C}$ & 109.5 \\
\hline $\mathrm{N} 1-\mathrm{O} 2-\mathrm{Na} 1$ & $96.34(11)$ & $\mathrm{H} 18 \mathrm{~A}-\mathrm{C} 18-\mathrm{H} 18 \mathrm{C}$ & 109.5 \\
\hline $\mathrm{C} 19-\mathrm{O} 4-\mathrm{Na} 1$ & $108.10(13)$ & $\mathrm{H} 18 \mathrm{~B}-\mathrm{C} 18-\mathrm{H} 18 \mathrm{C}$ & 109.5 \\
\hline $\mathrm{C} 19-\mathrm{O} 4-\mathrm{H} 4 \mathrm{O}$ & 108.2 & $\mathrm{O} 4-\mathrm{C} 19-\mathrm{Na} 1$ & $46.47(10)$ \\
\hline $\mathrm{Na} 1-\mathrm{O} 4-\mathrm{H} 4 \mathrm{O}$ & 119.0 & $\mathrm{O} 4-\mathrm{C} 19-\mathrm{H} 19 \mathrm{~A}$ & 109.5 \\
\hline $\mathrm{C} 6-\mathrm{O} 5-\mathrm{Ni} 1$ & $127.75(10)$ & $\mathrm{Na} 1-\mathrm{C} 19-\mathrm{H} 19 \mathrm{~A}$ & 79.9 \\
\hline $\mathrm{C} 6-\mathrm{O} 5-\mathrm{Na} 1$ & $125.60(10)$ & $\mathrm{O} 4-\mathrm{C} 19-\mathrm{H} 19 \mathrm{~B}$ & 109.5 \\
\hline $\mathrm{Ni} 1-\mathrm{O} 5-\mathrm{Na} 1$ & $106.12(5)$ & $\mathrm{Na} 1-\mathrm{C} 19-\mathrm{H} 19 \mathrm{~B}$ & 155.4 \\
\hline $\mathrm{C} 16-\mathrm{O} 6-\mathrm{Ni1}$ & $127.73(10)$ & $\mathrm{H} 19 \mathrm{~A}-\mathrm{C} 19-\mathrm{H} 19 \mathrm{~B}$ & 109.5 \\
\hline $\mathrm{C} 16-\mathrm{O} 6-\mathrm{Na} 1$ & $123.96(9)$ & $\mathrm{O} 4-\mathrm{C} 19-\mathrm{H} 19 \mathrm{C}$ & 109.5 \\
\hline $\mathrm{Ni1}-\mathrm{O} 6-\mathrm{Na} 1$ & $107.75(5)$ & $\mathrm{Na} 1-\mathrm{C} 19-\mathrm{H} 19 \mathrm{C}$ & 87.4 \\
\hline $\mathrm{C} 15-\mathrm{O} 7-\mathrm{C} 17$ & $118.69(13)$ & $\mathrm{H} 19 \mathrm{~A}-\mathrm{C} 19-\mathrm{H} 19 \mathrm{C}$ & 109.5 \\
\hline $\mathrm{C} 15-\mathrm{O} 7-\mathrm{Na} 1$ & $118.60(9)$ & $\mathrm{H} 19 \mathrm{~B}-\mathrm{C} 19-\mathrm{H} 19 \mathrm{C}$ & 109.5 \\
\hline $\mathrm{C} 17-\mathrm{O} 7-\mathrm{Na} 1$ & $122.69(10)$ & $\mathrm{O} 6-\mathrm{Ni} 1-\mathrm{N} 3$ & $95.02(5)$ \\
\hline $\mathrm{C} 1-\mathrm{O} 8-\mathrm{C} 18$ & $118.12(13)$ & $\mathrm{O} 6-\mathrm{Ni} 1-\mathrm{O} 5$ & $83.21(5)$ \\
\hline $\mathrm{C} 1-\mathrm{O} 8-\mathrm{Na} 1$ & $118.85(9)$ & $\mathrm{N} 3-\mathrm{Ni} 1-\mathrm{O} 5$ & $178.04(5)$ \\
\hline $\mathrm{C} 18-\mathrm{O} 8-\mathrm{Na} 1$ & $120.66(11)$ & $\mathrm{O} 6-\mathrm{Ni} 1-\mathrm{N} 2$ & $177.63(6)$ \\
\hline $\mathrm{O} 8-\mathrm{C} 1-\mathrm{C} 2$ & $125.03(16)$ & $\mathrm{N} 3-\mathrm{Ni1}-\mathrm{N} 2$ & $87.08(6)$ \\
\hline $\mathrm{O} 8-\mathrm{C} 1-\mathrm{C} 6$ & $113.83(13)$ & $\mathrm{O} 5-\mathrm{Ni} 1-\mathrm{N} 2$ & $94.71(6)$ \\
\hline $\mathrm{C} 2-\mathrm{C} 1-\mathrm{C} 6$ & $121.13(16)$ & $\mathrm{O} 6-\mathrm{Ni} 1-\mathrm{Na} 1$ & $41.09(3)$ \\
\hline $\mathrm{C} 1-\mathrm{C} 2-\mathrm{C} 3$ & $119.59(18)$ & $\mathrm{N} 3-\mathrm{Ni} 1-\mathrm{Na} 1$ & $135.99(4)$ \\
\hline $\mathrm{C} 1-\mathrm{C} 2-\mathrm{H} 2$ & 120.2 & $\mathrm{O} 5-\mathrm{Ni} 1-\mathrm{Na} 1$ & $42.30(3)$ \\
\hline $\mathrm{C} 3-\mathrm{C} 2-\mathrm{H} 2$ & 120.2 & $\mathrm{~N} 2-\mathrm{Ni} 1-\mathrm{Na} 1$ & $136.74(5)$ \\
\hline $\mathrm{C} 4-\mathrm{C} 3-\mathrm{C} 2$ & $120.82(16)$ & $\mathrm{O} 6-\mathrm{Na} 1-\mathrm{O} 5$ & $62.62(4)$ \\
\hline $\mathrm{C} 4-\mathrm{C} 3-\mathrm{H} 3$ & 119.6 & $\mathrm{O} 6-\mathrm{Na} 1-\mathrm{O} 4$ & $105.64(6)$ \\
\hline $\mathrm{C} 2-\mathrm{C} 3-\mathrm{H} 3$ & 119.6 & $\mathrm{O} 5-\mathrm{Na} 1-\mathrm{O} 4$ & $102.21(5)$ \\
\hline $\mathrm{C} 3-\mathrm{C} 4-\mathrm{C} 5$ & $121.15(18)$ & $\mathrm{O} 6-\mathrm{Na} 1-\mathrm{O} 7$ & $64.88(4)$ \\
\hline $\mathrm{C} 3-\mathrm{C} 4-\mathrm{H} 4$ & 119.4 & $\mathrm{O} 5-\mathrm{Na} 1-\mathrm{O} 7$ & $127.21(5)$ \\
\hline $\mathrm{C} 5-\mathrm{C} 4-\mathrm{H} 4$ & 119.4 & $\mathrm{O} 4-\mathrm{Na} 1-\mathrm{O} 7$ & $86.56(5)$ \\
\hline $\mathrm{C} 6-\mathrm{C} 5-\mathrm{C} 7$ & $121.30(15)$ & $\mathrm{O} 6-\mathrm{Na} 1-\mathrm{O} 2$ & $139.36(6)$ \\
\hline $\mathrm{C} 6-\mathrm{C} 5-\mathrm{C} 4$ & $118.75(17)$ & $\mathrm{O} 5-\mathrm{Na} 1-\mathrm{O} 2$ & $136.66(5)$ \\
\hline $\mathrm{C} 7-\mathrm{C} 5-\mathrm{C} 4$ & $119.95(16)$ & $\mathrm{O} 4-\mathrm{Na} 1-\mathrm{O} 2$ & $103.03(6)$ \\
\hline $\mathrm{O} 5-\mathrm{C} 6-\mathrm{C} 5$ & $123.93(15)$ & $\mathrm{O} 7-\mathrm{Na} 1-\mathrm{O} 2$ & $88.99(5)$ \\
\hline $\mathrm{O} 5-\mathrm{C} 6-\mathrm{C} 1$ & $117.52(14)$ & $\mathrm{O} 6-\mathrm{Na} 1-\mathrm{O} 8$ & $125.89(5)$ \\
\hline $\mathrm{C} 5-\mathrm{C} 6-\mathrm{C} 1$ & $118.55(14)$ & $\mathrm{O} 5-\mathrm{Na} 1-\mathrm{O} 8$ & $63.39(4)$ \\
\hline $\mathrm{N} 2-\mathrm{C} 7-\mathrm{C} 5$ & $125.72(15)$ & $\mathrm{O} 4-\mathrm{Na} 1-\mathrm{O} 8$ & $82.22(5)$ \\
\hline $\mathrm{N} 2-\mathrm{C} 7-\mathrm{H} 7$ & 117.1 & $\mathrm{O} 7-\mathrm{Na} 1-\mathrm{O} 8$ & $166.29(5)$ \\
\hline $\mathrm{C} 5-\mathrm{C} 7-\mathrm{H} 7$ & 117.1 & $\mathrm{O} 2-\mathrm{Na} 1-\mathrm{O} 8$ & $85.91(5)$ \\
\hline
\end{tabular}




\begin{tabular}{|c|c|c|c|}
\hline $\mathrm{N} 2-\mathrm{C} 8-\mathrm{C} 9$ & $109.13(14)$ & $\mathrm{O} 6-\mathrm{Na} 1-\mathrm{O} 1$ & $102.51(5)$ \\
\hline $\mathrm{N} 2-\mathrm{C} 8-\mathrm{H} 8 \mathrm{~A}$ & 109.9 & $\mathrm{O} 5-\mathrm{Na} 1-\mathrm{O} 1$ & $95.38(5)$ \\
\hline $\mathrm{C} 9-\mathrm{C} 8-\mathrm{H} 8 \mathrm{~A}$ & 109.9 & $\mathrm{O} 4-\mathrm{Na} 1-\mathrm{O} 1$ & $151.34(6)$ \\
\hline $\mathrm{N} 2-\mathrm{C} 8-\mathrm{H} 8 \mathrm{~B}$ & 109.9 & $\mathrm{O} 7-\mathrm{Na} 1-\mathrm{O} 1$ & $100.68(6)$ \\
\hline $\mathrm{C} 9-\mathrm{C} 8-\mathrm{H} 8 \mathrm{~B}$ & 109.9 & $\mathrm{O} 2-\mathrm{Na} 1-\mathrm{O} 1$ & $50.02(5)$ \\
\hline $\mathrm{H} 8 \mathrm{~A}-\mathrm{C} 8-\mathrm{H} 8 \mathrm{~B}$ & 108.3 & $\mathrm{O} 8-\mathrm{Na} 1-\mathrm{O} 1$ & $85.75(6)$ \\
\hline $\mathrm{N} 3-\mathrm{C} 9-\mathrm{C} 8$ & $109.49(14)$ & $\mathrm{O} 6-\mathrm{Na} 1-\mathrm{N} 1$ & $124.94(5)$ \\
\hline N3-C9-H9A & 109.8 & $\mathrm{O} 5-\mathrm{Na} 1-\mathrm{N} 1$ & $114.82(5)$ \\
\hline $\mathrm{C} 8-\mathrm{C} 9-\mathrm{H} 9 \mathrm{~A}$ & 109.8 & $\mathrm{O} 4-\mathrm{Na} 1-\mathrm{N} 1$ & $126.53(6)$ \\
\hline $\mathrm{N} 3-\mathrm{C} 9-\mathrm{H} 9 \mathrm{~B}$ & 109.8 & $\mathrm{O} 7-\mathrm{Na} 1-\mathrm{N} 1$ & $98.99(5)$ \\
\hline $\mathrm{C} 8-\mathrm{C} 9-\mathrm{H} 9 \mathrm{~B}$ & 109.8 & $\mathrm{O} 2-\mathrm{Na} 1-\mathrm{N} 1$ & $25.31(4)$ \\
\hline $\mathrm{H} 9 \mathrm{~A}-\mathrm{C} 9-\mathrm{H} 9 \mathrm{~B}$ & 108.2 & $\mathrm{O} 8-\mathrm{Na} 1-\mathrm{N} 1$ & $81.61(5)$ \\
\hline $\mathrm{N} 3-\mathrm{C} 10-\mathrm{C} 11$ & $125.54(14)$ & $\mathrm{O} 1-\mathrm{Na} 1-\mathrm{N} 1$ & $25.25(4)$ \\
\hline $\mathrm{N} 3-\mathrm{C} 10-\mathrm{H} 10$ & 117.2 & $\mathrm{O} 6-\mathrm{Na} 1-\mathrm{C} 19$ & $88.26(6)$ \\
\hline $\mathrm{C} 11-\mathrm{C} 10-\mathrm{H} 10$ & 117.2 & $\mathrm{O} 5-\mathrm{Na} 1-\mathrm{C} 19$ & $77.74(6)$ \\
\hline $\mathrm{C} 16-\mathrm{C} 11-\mathrm{C} 12$ & $119.08(15)$ & $\mathrm{O} 4-\mathrm{Na} 1-\mathrm{C} 19$ & $25.43(6)$ \\
\hline $\mathrm{C} 16-\mathrm{C} 11-\mathrm{C} 10$ & $120.98(14)$ & $\mathrm{O} 7-\mathrm{Na} 1-\mathrm{C} 19$ & $95.80(6)$ \\
\hline $\mathrm{C} 12-\mathrm{C} 11-\mathrm{C} 10$ & $119.84(14)$ & $\mathrm{O} 2-\mathrm{Na} 1-\mathrm{C} 19$ & $126.74(6)$ \\
\hline $\mathrm{C} 13-\mathrm{C} 12-\mathrm{C} 11$ & $120.74(15)$ & $\mathrm{O} 8-\mathrm{Na} 1-\mathrm{C} 19$ & $77.25(6)$ \\
\hline $\mathrm{C} 13-\mathrm{C} 12-\mathrm{H} 12$ & 119.6 & $\mathrm{O} 1-\mathrm{Na} 1-\mathrm{C} 19$ & $162.98(6)$ \\
\hline $\mathrm{C} 11-\mathrm{C} 12-\mathrm{H} 12$ & 119.6 & $\mathrm{~N} 1-\mathrm{Na} 1-\mathrm{C} 19$ & $146.80(6)$ \\
\hline $\mathrm{C} 12-\mathrm{C} 13-\mathrm{C} 14$ & $121.20(15)$ & $\mathrm{O} 6-\mathrm{Na} 1-\mathrm{Ni} 1$ & $31.17(3)$ \\
\hline $\mathrm{C} 12-\mathrm{C} 13-\mathrm{H} 13$ & 119.4 & $\mathrm{O} 5-\mathrm{Na} 1-\mathrm{Ni} 1$ & $31.58(3)$ \\
\hline $\mathrm{C} 14-\mathrm{C} 13-\mathrm{H} 13$ & 119.4 & $\mathrm{O} 4-\mathrm{Na} 1-\mathrm{Ni} 1$ & $108.42(5)$ \\
\hline $\mathrm{C} 15-\mathrm{C} 14-\mathrm{C} 13$ & $119.21(16)$ & $\mathrm{O} 7-\mathrm{Na} 1-\mathrm{Ni} 1$ & $96.03(3)$ \\
\hline $\mathrm{C} 15-\mathrm{C} 14-\mathrm{H} 14$ & 120.4 & $\mathrm{O} 2-\mathrm{Na} 1-\mathrm{Ni} 1$ & $148.38(4)$ \\
\hline $\mathrm{C} 13-\mathrm{C} 14-\mathrm{H} 14$ & 120.4 & $\mathrm{O} 8-\mathrm{Na} 1-\mathrm{Ni} 1$ & $94.97(3)$ \\
\hline $\mathrm{O} 7-\mathrm{C} 15-\mathrm{C} 14$ & $125.11(15)$ & $\mathrm{O} 1-\mathrm{Na} 1-\mathrm{Ni} 1$ & $98.44(4)$ \\
\hline $\mathrm{O} 7-\mathrm{C} 15-\mathrm{C} 16$ & $113.90(12)$ & $\mathrm{N} 1-\mathrm{Na} 1-\mathrm{Ni} 1$ & $123.52(4)$ \\
\hline $\mathrm{C} 14-\mathrm{C} 15-\mathrm{C} 16$ & $120.99(15)$ & $\mathrm{C} 19-\mathrm{Na} 1-\mathrm{Ni} 1$ & $83.90(5)$ \\
\hline $\mathrm{O} 3-\mathrm{N} 1-\mathrm{O} 1-\mathrm{Na} 1$ & $164.40(14)$ & $\mathrm{C} 12-\mathrm{C} 13-\mathrm{C} 14-\mathrm{C} 15$ & $0.3(3)$ \\
\hline $\mathrm{O} 2-\mathrm{N} 1-\mathrm{O} 1-\mathrm{Na} 1$ & $-15.51(17)$ & $\mathrm{C} 17-\mathrm{O} 7-\mathrm{C} 15-\mathrm{C} 14$ & $6.0(3)$ \\
\hline $\mathrm{O} 3-\mathrm{N} 1-\mathrm{O} 2-\mathrm{Na} 1$ & $-163.87(15)$ & $\mathrm{Na} 1-\mathrm{O} 7-\mathrm{C} 15-\mathrm{C} 14$ & $-172.37(14)$ \\
\hline $\mathrm{O} 1-\mathrm{N} 1-\mathrm{O} 2-\mathrm{Na} 1$ & $16.04(18)$ & $\mathrm{C} 17-\mathrm{O} 7-\mathrm{C} 15-\mathrm{C} 16$ & $-173.20(18)$ \\
\hline $\mathrm{C} 18-\mathrm{O} 8-\mathrm{C} 1-\mathrm{C} 2$ & $9.2(3)$ & $\mathrm{Na} 1-\mathrm{O} 7-\mathrm{C} 15-\mathrm{C} 16$ & $8.43(19)$ \\
\hline $\mathrm{Na} 1-\mathrm{O} 8-\mathrm{C} 1-\mathrm{C} 2$ & $171.84(14)$ & $\mathrm{C} 13-\mathrm{C} 14-\mathrm{C} 15-\mathrm{O} 7$ & $-179.79(16)$ \\
\hline $\mathrm{C} 18-\mathrm{O} 8-\mathrm{C} 1-\mathrm{C} 6$ & $-169.82(18)$ & $\mathrm{C} 13-\mathrm{C} 14-\mathrm{C} 15-\mathrm{C} 16$ & $-0.6(3)$ \\
\hline $\mathrm{Na} 1-\mathrm{O} 8-\mathrm{C} 1-\mathrm{C} 6$ & $-7.19(19)$ & $\mathrm{Ni1}-\mathrm{O} 6-\mathrm{C} 16-\mathrm{C} 11$ & $0.5(2)$ \\
\hline $\mathrm{O} 8-\mathrm{C} 1-\mathrm{C} 2-\mathrm{C} 3$ & $-179.16(17)$ & $\mathrm{Na} 1-\mathrm{O} 6-\mathrm{C} 16-\mathrm{C} 11$ & $170.80(12)$ \\
\hline $\mathrm{C} 6-\mathrm{C} 1-\mathrm{C} 2-\mathrm{C} 3$ & $-0.2(3)$ & $\mathrm{Ni1}-\mathrm{O} 6-\mathrm{C} 16-\mathrm{C} 15$ & $-179.59(11)$ \\
\hline $\mathrm{C} 1-\mathrm{C} 2-\mathrm{C} 3-\mathrm{C} 4$ & $-0.6(3)$ & $\mathrm{Na} 1-\mathrm{O} 6-\mathrm{C} 16-\mathrm{C} 15$ & $-9.3(2)$ \\
\hline $\mathrm{C} 2-\mathrm{C} 3-\mathrm{C} 4-\mathrm{C} 5$ & $0.6(3)$ & $\mathrm{C} 12-\mathrm{C} 11-\mathrm{C} 16-\mathrm{O} 6$ & $179.76(15)$ \\
\hline $\mathrm{C} 3-\mathrm{C} 4-\mathrm{C} 5-\mathrm{C} 6$ & $0.1(3)$ & $\mathrm{C} 10-\mathrm{C} 11-\mathrm{C} 16-\mathrm{O} 6$ & $3.2(3)$ \\
\hline $\mathrm{C} 3-\mathrm{C} 4-\mathrm{C} 5-\mathrm{C} 7$ & $178.98(19)$ & $\mathrm{C} 12-\mathrm{C} 11-\mathrm{C} 16-\mathrm{C} 15$ & $-0.2(2)$ \\
\hline $\mathrm{Ni} 1-\mathrm{O} 5-\mathrm{C} 6-\mathrm{C} 5$ & $-1.7(2)$ & $\mathrm{C} 10-\mathrm{C} 11-\mathrm{C} 16-\mathrm{C} 15$ & $-176.70(15)$ \\
\hline $\mathrm{Na} 1-\mathrm{O} 5-\mathrm{C} 6-\mathrm{C} 5$ & $-172.10(12)$ & $\mathrm{O} 7-\mathrm{C} 15-\mathrm{C} 16-\mathrm{O} 6$ & $-0.1(2)$ \\
\hline $\mathrm{Ni} 1-\mathrm{O} 5-\mathrm{C} 6-\mathrm{C} 1$ & $177.62(12)$ & $\mathrm{C} 14-\mathrm{C} 15-\mathrm{C} 16-\mathrm{O} 6$ & $-179.34(15)$ \\
\hline
\end{tabular}




\begin{tabular}{|c|c|c|c|}
\hline $\mathrm{Na} 1-\mathrm{O} 5-\mathrm{C} 6-\mathrm{C} 1$ & $7.2(2)$ & $\mathrm{O} 7-\mathrm{C} 15-\mathrm{C} 16-\mathrm{C} 11$ & $179.82(15)$ \\
\hline $\mathrm{C} 7-\mathrm{C} 5-\mathrm{C} 6-\mathrm{O} 5$ & $-0.5(3)$ & $\mathrm{C} 14-\mathrm{C} 15-\mathrm{C} 16-\mathrm{C} 11$ & $0.6(2)$ \\
\hline $\mathrm{C} 4-\mathrm{C} 5-\mathrm{C} 6-\mathrm{O} 5$ & $178.43(16)$ & $\mathrm{C} 16-\mathrm{O} 6-\mathrm{Ni} 1-\mathrm{N} 3$ & $-4.68(14)$ \\
\hline $\mathrm{C} 7-\mathrm{C} 5-\mathrm{C} 6-\mathrm{C} 1$ & $-179.72(16)$ & $\mathrm{Na} 1-\mathrm{O} 6-\mathrm{Ni} 1-\mathrm{N} 3$ & $-176.25(6)$ \\
\hline $\mathrm{C} 4-\mathrm{C} 5-\mathrm{C} 6-\mathrm{C} 1$ & $-0.8(3)$ & $\mathrm{C} 16-\mathrm{O} 6-\mathrm{Ni1}-\mathrm{O} 5$ & $176.15(14)$ \\
\hline $\mathrm{O} 8-\mathrm{C} 1-\mathrm{C} 6-\mathrm{O} 5$ & $0.7(2)$ & $\mathrm{Na} 1-\mathrm{O} 6-\mathrm{Ni1}-\mathrm{O} 5$ & $4.58(6)$ \\
\hline $\mathrm{C} 2-\mathrm{C} 1-\mathrm{C} 6-\mathrm{O} 5$ & $-178.41(16)$ & $\mathrm{C} 16-\mathrm{O} 6-\mathrm{Ni} 1-\mathrm{Na} 1$ & $171.57(17)$ \\
\hline $\mathrm{O} 8-\mathrm{C} 1-\mathrm{C} 6-\mathrm{C} 5$ & $179.98(15)$ & $\mathrm{C} 10-\mathrm{N} 3-\mathrm{Ni} 1-\mathrm{O} 6$ & $7.18(15)$ \\
\hline $\mathrm{C} 2-\mathrm{C} 1-\mathrm{C} 6-\mathrm{C} 5$ & $0.9(3)$ & $\mathrm{C} 9-\mathrm{N} 3-\mathrm{Ni} 1-\mathrm{O} 6$ & $-171.60(13)$ \\
\hline $\mathrm{C} 8-\mathrm{N} 2-\mathrm{C} 7-\mathrm{C} 5$ & $174.90(17)$ & $\mathrm{C} 10-\mathrm{N} 3-\mathrm{Ni} 1-\mathrm{N} 2$ & $-171.71(15)$ \\
\hline $\mathrm{Ni} 1-\mathrm{N} 2-\mathrm{C} 7-\mathrm{C} 5$ & $0.6(3)$ & $\mathrm{C} 9-\mathrm{N} 3-\mathrm{Ni} 1-\mathrm{N} 2$ & $9.50(13)$ \\
\hline $\mathrm{C} 6-\mathrm{C} 5-\mathrm{C} 7-\mathrm{N} 2$ & $1.0(3)$ & $\mathrm{C} 10-\mathrm{N} 3-\mathrm{Ni} 1-\mathrm{Na} 1$ & $3.64(18)$ \\
\hline $\mathrm{C} 4-\mathrm{C} 5-\mathrm{C} 7-\mathrm{N} 2$ & $-177.89(17)$ & $\mathrm{C} 9-\mathrm{N} 3-\mathrm{Ni} 1-\mathrm{Na} 1$ & $-175.15(10)$ \\
\hline $\mathrm{C} 7-\mathrm{N} 2-\mathrm{C} 8-\mathrm{C} 9$ & $168.53(17)$ & $\mathrm{C} 6-\mathrm{O} 5-\mathrm{Ni} 1-\mathrm{O} 6$ & $-176.40(15)$ \\
\hline $\mathrm{Ni} 1-\mathrm{N} 2-\mathrm{C} 8-\mathrm{C} 9$ & $-16.6(2)$ & $\mathrm{Na} 1-\mathrm{O} 5-\mathrm{Ni1}-\mathrm{O} 6$ & $-4.48(6)$ \\
\hline $\mathrm{C} 10-\mathrm{N} 3-\mathrm{C} 9-\mathrm{C} 8$ & $160.57(16)$ & $\mathrm{C} 6-\mathrm{O} 5-\mathrm{Ni} 1-\mathrm{N} 2$ & $2.46(15)$ \\
\hline $\mathrm{Ni} 1-\mathrm{N} 3-\mathrm{C} 9-\mathrm{C} 8$ & $-20.5(2)$ & $\mathrm{Na} 1-\mathrm{O} 5-\mathrm{Ni1}-\mathrm{N} 2$ & $174.39(6)$ \\
\hline $\mathrm{N} 2-\mathrm{C} 8-\mathrm{C} 9-\mathrm{N} 3$ & $22.7(2)$ & $\mathrm{C} 6-\mathrm{O} 5-\mathrm{Ni} 1-\mathrm{Na} 1$ & $-171.92(17)$ \\
\hline $\mathrm{C} 9-\mathrm{N} 3-\mathrm{C} 10-\mathrm{C} 11$ & $173.00(16)$ & $\mathrm{C} 7-\mathrm{N} 2-\mathrm{Ni} 1-\mathrm{N} 3$ & $178.85(16)$ \\
\hline $\mathrm{Ni1}-\mathrm{N} 3-\mathrm{C} 10-\mathrm{C} 11$ & $-5.7(3)$ & $\mathrm{C} 8-\mathrm{N} 2-\mathrm{Ni} 1-\mathrm{N} 3$ & $4.44(14)$ \\
\hline $\mathrm{N} 3-\mathrm{C} 10-\mathrm{C} 11-\mathrm{C} 16$ & $-0.5(3)$ & $\mathrm{C} 7-\mathrm{N} 2-\mathrm{Ni} 1-\mathrm{O} 5$ & $-1.95(16)$ \\
\hline $\mathrm{N} 3-\mathrm{C} 10-\mathrm{C} 11-\mathrm{C} 12$ & $-177.00(17)$ & $\mathrm{C} 8-\mathrm{N} 2-\mathrm{Ni} 1-\mathrm{O} 5$ & $-176.35(13)$ \\
\hline $\mathrm{C} 16-\mathrm{C} 11-\mathrm{C} 12-\mathrm{C} 13$ & $-0.2(3)$ & $\mathrm{C} 7-\mathrm{N} 2-\mathrm{Ni} 1-\mathrm{Na} 1$ & $3.57(19)$ \\
\hline $\mathrm{C} 10-\mathrm{C} 11-\mathrm{C} 12-\mathrm{C} 13$ & $176.39(16)$ & $\mathrm{C} 8-\mathrm{N} 2-\mathrm{Ni} 1-\mathrm{Na} 1$ & $-170.84(11)$ \\
\hline $\mathrm{C} 11-\mathrm{C} 12-\mathrm{C} 13-\mathrm{C} 14$ & $0.1(3)$ & & \\
\hline
\end{tabular}

Hydrogen-bond geometry $\left(\AA,{ }^{\circ}\right)$

\begin{tabular}{lllll}
\hline$D-\mathrm{H} \cdots A$ & $D-\mathrm{H}$ & $\mathrm{H} \cdots A$ & $D \cdots A$ & $D-\mathrm{H} \cdots A$ \\
\hline $\mathrm{O} 4-\mathrm{H} 4 O \cdots \mathrm{O} 1^{\mathrm{i}}$ & 0.82 & 2.24 & $2.991(2)$ & 154 \\
$\mathrm{O} 4-\mathrm{H} 4 O \cdots \mathrm{O} 3^{\mathrm{i}}$ & 0.82 & 2.49 & $3.181(2)$ & 143 \\
$\mathrm{C} 8-\mathrm{H} 8 B \cdots \mathrm{O} 2^{\mathrm{ii}}$ & 0.97 & 2.65 & $3.152(2)$ & 112 \\
\hline
\end{tabular}

Symmetry codes: (i) $x-1, y, z$; (ii) $x, y-1, z$. 